\title{
Coverage and Adaptive Scheduling Algorithms for Criticality Management on Video Wireless Sensor Networks
}

\author{
Abdallah Makhoul, Rachid Saadi and Congduc Pham \\ University of Pau, LIUPPA Laboratory \\ \{abdallah.makhoul, rachid.saadi, congduc.pham\}@univ-pau.fr \\ UFR Sciences et Techniques, Avenue de l'Université, BP : 1155, 64013 Pau CEDEX France
}

\begin{abstract}
In WSN-based surveillance systems power conservation is an important issue but one must also ensure that the application's objectives are met. In this paper, we jointly address the problem of coverage, disambigation and adaptive scheduling of sensor node's activity to extend the network lifetime while fulfilling a critical surveillance application needs. We first present a distributed algorithm that ensures both coverage of the deployment area and network connectivity by providing multiple cover sets to manage Field of View redundancies. The availability of multiple cover sets leads to a simple but efficient disambigation algorithm. Then, we propose a multiple levels activity model that uses behavior functions modeled by modified Bezier curves to define application classes and allow for adaptive scheduling. Simulation results are presented to validate the performance of the proposed approach.
\end{abstract}

\section{INTRODUCTION}

A Wireless Video Sensor Networks (WVSN) consists of a set of sensor nodes equipped with miniaturized video cameras. In this paper, we are more particularly interested in WVSN for surveillance applications. In these scenarios, hundreds or thousands of video nodes of low capacity (resolution, processing and storage) of a same or similar type can be deployed in an area of interest. These nodes would use collaboration mechanisms to ensure a surveillance task according to a given application and to transmit, via an ad-hoc network (mostly wireless), useful video data to one or more base stations. Desired features of such a surveillance infrastructure are high reliability and longest lifetime, largest coverage, disambiguation capabilities to name a few.

An important issue is how to ensure and maintain high coverage of the area of interest. The problem of coverage in many-robot system or WSN was largely studied and very interesting results were published. Most of the recent existing works on the connected coverage problem in scalar sensor networks [1], [2], [3], [4] typically assume omnidirectional sensors with disk-like sensing coverage. Thus, two scalar nodes are likely to be redundant if they are close to each other. However, in video sensor networks, video nodes possess limited sensing coverage area (sector coverage) due to the camera constraints and its Field of View (FoV). In addition, two video sensor nodes can be redundant and still be far from each other.

One other issue of prime importance is related to energy considerations since the scarcity of energy does have a direct impact on coverage as it is not possible to have all the sensor nodes in activity at the same time. In randomly deployed sensor networks, sensor nodes can be redundant (nodes that monitor the same region) leading to overlaps among the monitored areas. Therefore, a common approach is to define a subset of the deployed nodes to be active while the other nodes can sleep. The result is a schedule of the activity of sensor nodes in such a way that guarantees the deployment area coverage as well as the network connectivity. In addition, it is also desirable to be able to define multiple levels of activity that could correspond to how many samples are captured per unit of time. For instance some surveillance applications may focus on barrier coverage rather than blanket coverage. In this case, sensor nodes at the border of the area of interest should take more samples while interior nodes can decrease their activity. Also, multiple levels of activity may be simply necessary because surveillance applications such as an intrusion detection system has to be able to operate on a long term basis and because one does not know when such intrusions could occur. For video sensor networks efficient scheduling and the ability to provide multiple levels of activity is even more important than in traditional sensing systems (e.g. temperature, pressure,...) as capturing and transmitting images are much more energy-consuming. 
This paper presents a framework for adaptively schedule video sensor node's activity while addressing coverage, energy considerations and application's objectives. The final goal is to provide the necessary algorithmic support for a surveillance application to express its objectives. Regarding the coverage problem, our contribution is to propose a multiple cover set approach to manage FoV redundancies. The availability of multiple cover sets then allows us to propose a simple but efficient disambigation algorithm. Next, taking account the energy-considerations and the application's objectives, the framework seeks to provide multiple levels of activity by providing a default operating mode that links a node's capture rate to the size of its cover sets and two additional operating modes that force a node to be in "high" or "low" status according to the surveillance application's specific objectives. These operating modes uses behavior functions modeled by modified Bezier curves that could define application classes.

The rest of this paper is organized as follows. Section II presents the coverage and node scheduling propositions. Section III presents the adaptive model that uses behavior functions modeled by modified Bezier curves for providing the necessary flexibility to the end applications. Simulation results are presented in section IV before the conclusions.

\section{COVERAGE AND SENSOR NODES SCHEDUling}

The coverage problem for wireless video sensor networks can be categorized as:

- Known-Targets Coverage Problem, which seeks to determine a subset of connected video nodes that covers a given set of target-locations scattered in a 2D plane.

- Region-Coverage Problem, which seeks to find a subset of connected video nodes that ensures the coverage of the entire region of deployment in a 2D plane.

Most of the previous works have considered the known-targets coverage problem [5], [6], [7], [8], [9]. The objective is to ensure at all-time the coverage of some targets with known locations which are deployed in a two-dimensional plane. For example, the authors in [9] organize sensor nodes into mutually exclusive subsets that are activated successively, where the size of each subset is restricted and not all of the targets need to be covered by the sensors in one subset.

In [6], a directional sensor model is proposed, where a sensor is allowed to work in several directions. The idea behind this is to find a minimal set of directions that can cover the maximum number of targets. It is different from the approach described in [5] that aims to find a group of non-disjoint cover sets, each set covering all the targets to maximize the network lifetime.

Concerning the area coverage problem, the existing works focus on finding an efficient deployment pattern so that the average overlapping area of each sensor is bounded. The authors in [10] analyze new deployment strategies for satisfying some given coverage probability requirements with directional sensing models. A model of directed communications is introduced to ensure and repair the network connectivity.

Based on a rotatable directional sensing model, the authors in [11] present a method to deterministically estimate the amount of directional nodes for a given coverage rate. A sensing connected sub-graph accompanied with a convex hull method is introduced to model a directional sensor network into several parts in a distributed manner. With adjustable sensing directions, the coverage algorithm tries to minimize the overlapping sensing area of directional sensors only with local topology information.

Different from the above works, our paper mainly focuses on the area coverage problem and more specifically on scheduling for randomly deployed video sensor nodes. The local coverage objective of each sensor node is to ensure, at all time, the coverage of this area either by itself (if it is active) or by a subset of its neighbors, called the cover set.

\section{A. Coverage and node's cover set}

A video sensor node $v$ is represented by the FoV of its camera. We consider a 2-D model of a video sensor node where the FoV is defined as a sector denoted by a 4-tuple $v\left(P, R_{s}, \vec{V}, \alpha\right)$. Here $P$ is the position of $v$, $R_{s}$ is its sensing range, $\vec{V}$ is the vector representing the line of sight of the camera's FoV which determines the sensing direction, and $\alpha$ is the offset angle of the FoV on both sides of $\vec{V}$.

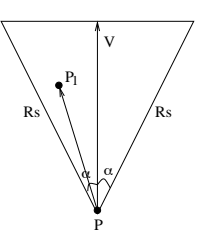

(a)

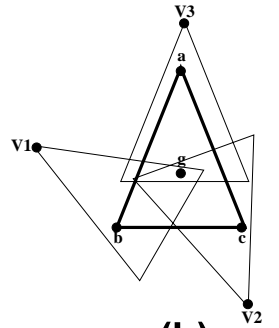

(b)

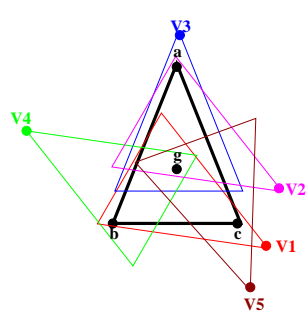

(c)
Fig. 1. Video sensing and coverage model

Figure 1a illustrates the FoV of a video sensor node in our model. In the reminder of this paper, we consider 
that all video nodes have the same characteristics: same sensing range $R_{s}$ and same offset angle $\alpha$.

The approach we described in this paper is completely distributed: every video node computes its local solution for coverage. Each node $v$ covers a sector area thanks to its FoV, which we call $v$ 's FoV area. Then, its local coverage objective is to ensure, at all time, the coverage of this area either by itself (if it is active) or by a subset of its neighbors. If every video node ensure that its local coverage objective is satisfied then global coverage is also satisfied.

Definition 1: We define a cover $C o_{i}(v)$ of a video node $v$ as a subset of video nodes such that: $\bigcup_{v^{\prime} \in C o_{i}(v)}\left(v^{\prime \prime}\right.$ s FoV area) covers $v$ 's FoV area.

Definition 2: $C o(v)$ is defined as the set of all the covers of node $v$.

An example of FoV coverage is shown in Figure 1b, where nodes $v_{1}, v_{2}$ and $v_{3}$ cover the FoV area of node $v$, represented by $a b c$. To compute $C o(v)$, our model uses four distinctive points: $a, b, c$ and $g$ (the center of gravity of $(a b c)$ ) to represent the FoV of $v$ as shown in Figure $1 b$. Then, we say that $v$ 's FoV is covered by a set $C o_{i}(v) \in C o(v)$ if:

1) $\forall v^{\prime} \in C o_{i}(v), v^{\prime}$ covers the point $g$ and at least one of the points $\{a, b, c\}$

2) $a, b, c$ and $g$ are covered by the elements of $C o_{i}(v)$.

In other terms, to compute $C o(v)$, a node $v$ finds the following sets where $N(v)$ represents the set of neighbors of node $v$ :

- $A=\left\{v^{\prime} \in N(v): v^{\prime}\right.$ covers point a of the FoV $\}$

- $B=\left\{v^{\prime} \in N(v): v^{\prime}\right.$ covers point $\mathbf{b}$ of the FoV $\}$

- $C=\left\{v^{\prime} \in N(v): v^{\prime}\right.$ covers point $\mathrm{c}$ of the FoV $\}$

- $G=\left\{v^{\prime} \in N(v): v^{\prime}\right.$ covers point $\mathrm{g}$ of the FoV $\}$

- $A G=\{A \cap G\}, B G=\{B \cap G\}, C G=\{C \cap G\}$

Then, $C o(v)$ is set to the cartesian product of sets $A G, B G$ and $C G(\{A G \times B G \times C G\})$. Note that the set-intersection function generates $n+m$ recursive calls in the worst case. Therefore, the intersection of 2 sets can be done with complexity of $O(n+m)$, where $m$ and $n$ are the cardinality of the two sets respectively. As the size of sets $A, B, C$ and $G$ is limited, a video node can easily computes the intersections.

In the example illustrated by Figure 1c, $v$ 's FoV is represented by $(a b c g)$. To find the set of covers, node $v$ finds the sets: $A G=\left\{v_{2}, v_{3}\right\}, B G=\left\{v_{1}, v_{4}\right\}$ and $C G=\left\{v_{1}, v_{5}\right\}$. Then, following the above method, the set of possible covers for $v$ is: $C o(v)=\left\{\{v\},\left\{v_{2}, v_{1}\right\}\right.$, $\left.\left\{v_{3}, v_{1}\right\},\left\{v_{2}, v_{4}, v_{5}\right\},\left\{v_{3}, v_{4}, v_{5}\right\}\right\}$.

This model allows a node $v$ to construct $C o(v)$ of its FoV area. Hence, in some cases, it can occur that a cover does not ensure the complete FoV coverage.
The effectiveness of such model will be shown in the experimental results section. Note that, one can consider more points such that the midpoints of segments [ab], [ac] and [bc]. On the other hand, doing so will reduce the number of covers and consequently increase the number of active nodes. Note that the way coverage is defined could be different. The method we use in this paper has the advantage of being simple since the main purpose here is to be able to construct cover sets.

\section{B. Scheduling nodes with cover sets}

The scheduling of video sensor nodes operates in 2 phases. The first is a setup phase where each node $v$ constructs its set of covers $C o(v)$. The second phase is the scheduling phase where each node decides to be active or in sleep mode. Our objective is to minimize the number of active nodes while ensuring the whole coverage area.

1) Construction of $C o(v)$ : At this step, each video node $v$ constructs all possible covers, $C o(v)$ that satisfy its local coverage objective (e.g. covering its FoV area). These sets are constructed by considering all the communication graph neighbors. Each node diffuses its position $P$ and its direction $\vec{V}$ to its neighbors and receives their informations. Each node constructs the sets $A, B$ and $C$ and then computes $C o_{i}(v)$ that overlap its FoV and ensure its coverage as explained in section II-A.

In the literature, most of existing omnidirectional sensing coverage works try to construct disjoint sets of active nodes [1], [2], [3], [4]. In our case, we have the possibility that two or more covers have some video nodes in common. This dependancy must be taken into account in the scheduling phase. Hence, selecting one cover also reduces the lifetime of the sensor it has in common with another cover. In this case we can consider the level of energy as a criteria while choosing the active cover. The energy level of the lowest node in $\mathrm{Co}_{i}(v)$ determines $\mathrm{Co}_{i}(v)$ 's energy level. We notice here that the singleton $\{v\}$ is considered as an element of the set $C o(v)$.

2) Video Node's Scheduling: The activity of video sensor nodes operates in rounds. For each round, every node decides to be active or not based on the activity messages received from its neighbors. At a first step, every node orders its sets of covers according to their cardinality, and gives priority to the covers with minimum cardinality and the lowest priority is given to the singleton $\{v\}$. After, during the network lifetime, the covers' priority order change according to the energy level of each cover. Note that another criteria can be defined. 
A video node $v$ receives the activity decisions of its neighbors. Then, it tests if the active nodes belong to a cover $C o_{i}(v)$ in which case $v$ goes in sleep mode after sending its decision to its neighbors. In the case where no $\mathrm{Co}_{i}(v)$ is satisfied, or because $v$ has the highest level of energy, $v$ decides to remain in active mode and diffuses its decision.

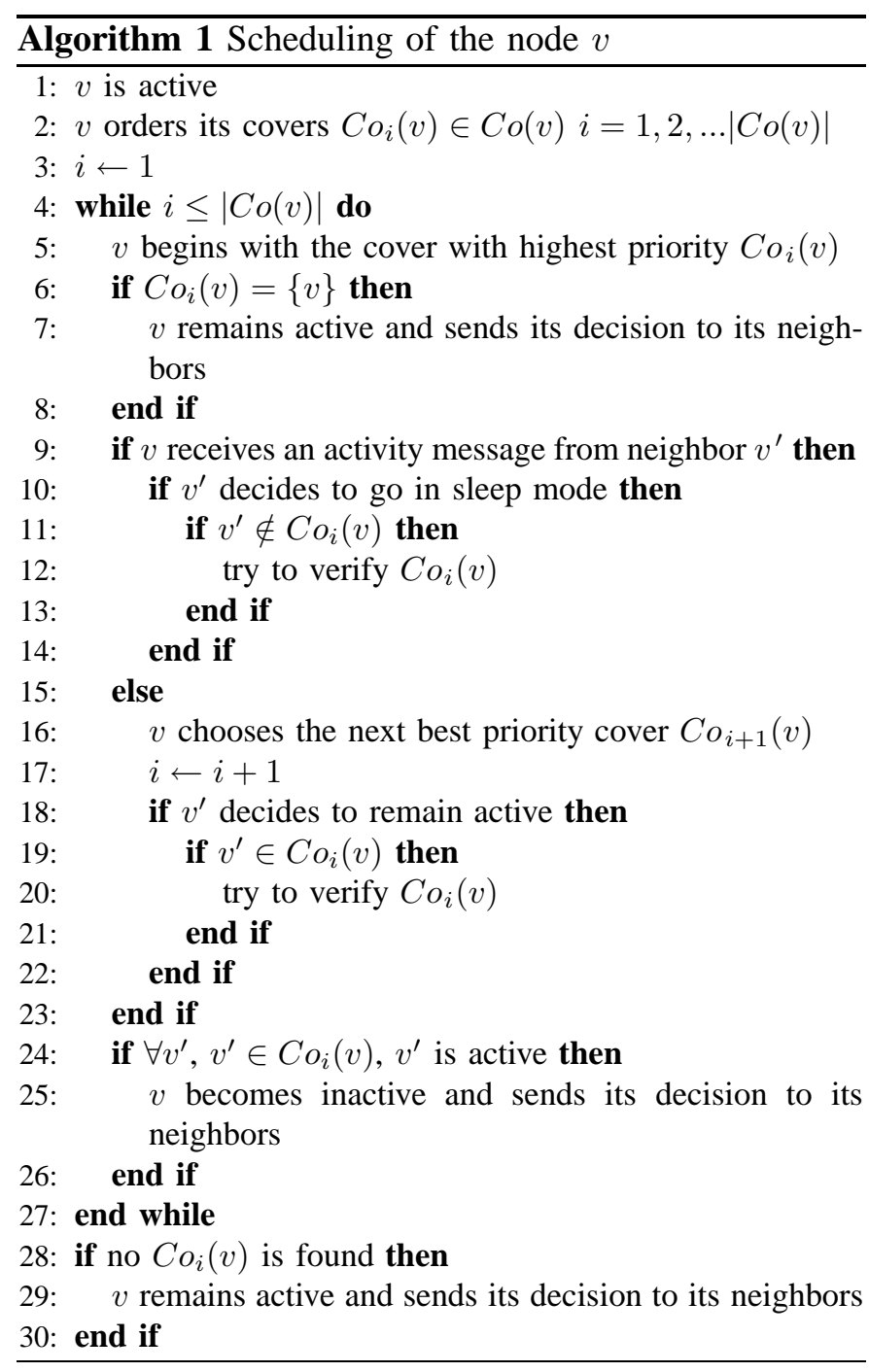

A video node $v$ orders the set $C o(v)$ according to their priorities (cardinality or energy level). Then, it starts with the first cover $C o_{1}(v) \in C o(v)$ (which has the highest priority) and tests if it is satisfied. If singleton $\{v\}$ has the highest priority it becomes active and difuuses its decision. A cover is comprised by a set of video nodes and if one node is or become inactive this cover can not be satisfied.

The node's scheduling process is summarized in Algorithm 1 . Node $v$ receives an activity message from its neighbor $v^{\prime}$, if $v^{\prime} \in C o_{1}(v)$ decided to be inactive, then $v$ goes to the next cover and so on until it finds an active cover or decides to be active itself. If node $v^{\prime} \in C o_{1}(v)$ is active then node $v$ check whether all nodes of $\mathrm{Co}_{1}(v)$ are in active mode in order to go in sleep mode. This process is repeated for each cover and at every round.

\section{ADAPTATION TO APPLICATION CRITICALITY}

For wireless video sensor nodes the frame capture process and transmission to a sink is a huge energyconsuming task. On the other hand, the higher the capture rate is, the better relevant events could be detected and identified. Therefore if we consider a video surveillance application for intrusion detection, video nodes should capture at a high rate due to the criticality of this type of application. There are other types of non-critical surveillance or monitoring applications where the capture rate does not need to be set to the maximum rate. However, even in the case of an intrusion detection application, it is not realistic to consider that video nodes should always capture at their maximum rate when in active mode because network lifetime is almost as important as coverage in such applications. In general, it is desirable to be able to adjust the capture rate according to the application's requirements. In our approach we express the application criticality by the $r^{0}$ variable which can take values between 0 and a defined 1 representing the low and the high criticality level respectively. Low level criticality indicates that the application does not require a high video frame capture rate while a high level criticality does. Then, according to the application's requirements, $r^{0}$ could be initialized accordingly into all sensors nodes prior to deployment.

\section{A. Static capture model}

A naïve approach would consist in fixing the frame capture rate of all video nodes to a given rate. For instance, high level criticality pushes video nodes to capture at near the maximum frame rate capability. However, this simple approach presents some drawbacks. In fact, (i) setting video nodes to work at full capacity provides very good capture quality but the network lifetime is very short, (ii) although setting the nodes at low capacity saves energy and extends the network lifetime, it provides poor surveillance quality, (iii) choosing a moderate frame capture rate could balance between capture quality and network lifetime but at the same time sensors can not be fully exploited if it is necessary.

\section{B. Dynamic capture model}

To fully exploit the video node capabilities we propose that a video node captures frames at a rate that is defined by the size of its cover set. Obviously, when a node 
has several covers (see definition 1) it can increase its frame capture rate because if it runs out of energy it can be replaced by one of its covers. On the other hand, the frame capture rate variation should depend on the criticality level of the application as discussed previously. In what follows we will define different application classes which will determine a node's frame capture rate.

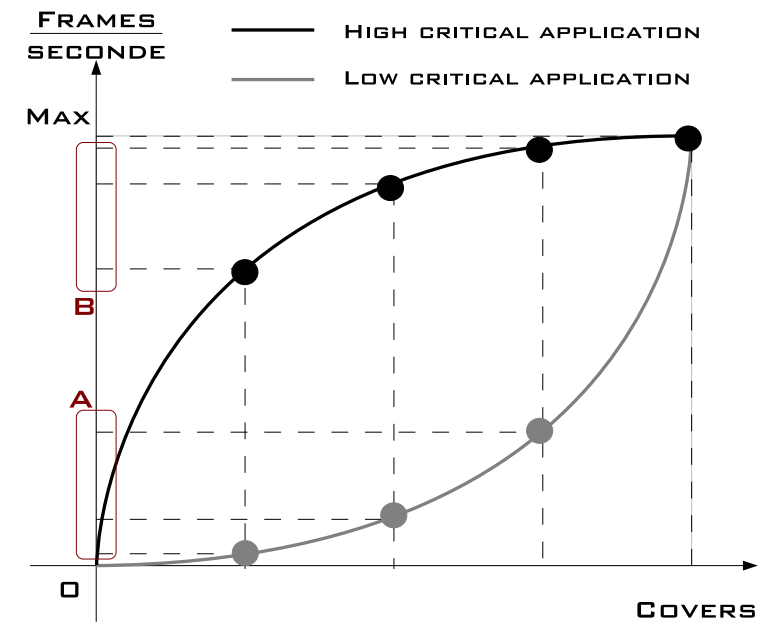

Fig. 2. Dynamic approach.

1) Application classes: We can broadly classify applications into different categories based on their criticality level. In our approach we define two classes of applications: high and low criticality applications. This criticality level is represented by a mathematical function $y=f_{r^{0}}(x)$ that we call BV (BehaVior) function. This function can oscillate from hyperbolic to parabolic shape as illustrated in Figure 2:

- values on the $x$ axis are positive integers representing the cardinality of the cover set $|C o|$. Integer values lie between 1 and $\max$, where $\max$ is fixed according to the network topology. A video sensor node that is the only node capable of covering its FoV has a cover size of 1 on the $x$ axis.

- the $y$ axis gives the corresponding frame capture rate based on the cardinality of the cover set expressed on the $x$ axis and the application criticality level $\left(r^{0}\right)$.

We now present the contrast between applications that exhibit high and low criticality level in terms of the BV function.

1) Class 1 "low criticality", $0 \leq r^{0}<0.5$ : this class of applications does not need high frame capture rate. This characteristic is represented by an hyperbolic BV function. As illustrated in figure 2 (box A), most projections of $x$ values are gathered close to zero (i.e. the majority of the sensors will preserve their energy by capturing slowly).

2) Class 2 "high criticality", $0.5 \leq r^{0} \leq 1$ : This class of applications needs high frame capture rate. This characteristic is represented by a parabolic BV function. As illustrated in figure 2 (box B), most projections of $x$ values are gathered close to the $\max$ frame capture rate (i.e. the majority of nodes capture at a high rate).

2) The behavior function: We use Bezier curve to model the BV function. Bezier curves are flexible and can plot easily a wide range of geometric curves and have successfully been used for modeling subjectivity in trust evaluation models in pervasive environments [12].

Definition 3: The bezier curve is a parametric form to draw a smooth curve. It is fulfilled through some points $P_{0}, P_{1} \ldots P_{n}$, starting at $P_{0}$ going towards $P_{1} \ldots P_{n-1}$ and terminating at $P_{n}$.

In our model we will use a Bezier curve with three points which is called a Quadratic Bezier curve. It is defined as follows:

Definition 4: A quadratic Bezier curve is the path traced by the function $\mathrm{B}(\mathrm{t})$, given points $P_{0}, P_{1}$, and $P_{2}$.

$$
B(t)=(1-t)^{2} * P_{0}+2 t(1-t) * P_{1}+t^{2} * P_{2} .
$$

The BV function is expressed by a Bezier curve that passes through three points:

- The origin point $\left(P_{0}(0,0)\right)$.

- The behavior point $\left(P_{1}\left(b_{x}, b_{y}\right)\right)$

- The threshold point $\left(P_{2}\left(h_{x}, h_{y}\right)\right)$ where $h_{x}$ represents the highest cover cardinality and $h_{y}$ represents the maximum frame capture rate determined by the sensor node hardware capabilities.

As illustrated in Figure 3, by moving the behavior point $P_{1}$ inside the rectangle defined by $P_{0}$ and $P_{2}$, we are able to adjust the curvature of the Bezier curve. The BV function describes the application criticality. It takes $|C o|$ as input on the $x$ axis and returns the corresponding "frame capture rate" on the $y$ axis. To apply the BV function with the Bezier curve, we modify this latter to obtain $y$ as a function of $x$, instead of taking a temporal variable $t$ as input to compute $x$ and $y$. Based on the Bezier curve, let us now define the "BV function":

Definition 5: The BV function curve can be drawn through the three points $P_{0}(0,0), P_{1}\left(b_{x}, b_{y}\right)$ and $P_{2}\left(h_{x}, h_{y}\right)$ using the Bezier curve as follows: 


$$
\begin{aligned}
& \begin{aligned}
B V:\left[0, h_{x}\right] & \longrightarrow\left[0, h_{y}\right] \\
X & \longrightarrow Y
\end{aligned} \\
& B V_{P_{1}, P_{2}}(X)= \\
& \begin{cases}\frac{\left(h_{y}-2 b_{y}\right)}{4 b_{x}^{2}} X^{2}+\frac{b_{y}}{b_{x}} X & \text { if }\left(h_{x}-2 b_{x}=0\right) \\
\left(h_{y}-2 b_{y}\right)(\propto(X))^{2}+2 b_{y} \propto(X), & \text { if }\left(h_{x}-2 b_{x} \neq 0\right)\end{cases} \\
& \text { Where } \propto(X)=\frac{-b_{x}+\sqrt{b_{x}^{2}-2 b_{x} * X+h_{x} * X}}{h_{x}-2 b_{x}} \wedge\left\{\begin{array}{l}
0 \leq b_{x} \leq h_{x} \\
0 \leq X \leq h_{x} \\
h_{x}>0
\end{array}\right.
\end{aligned}
$$

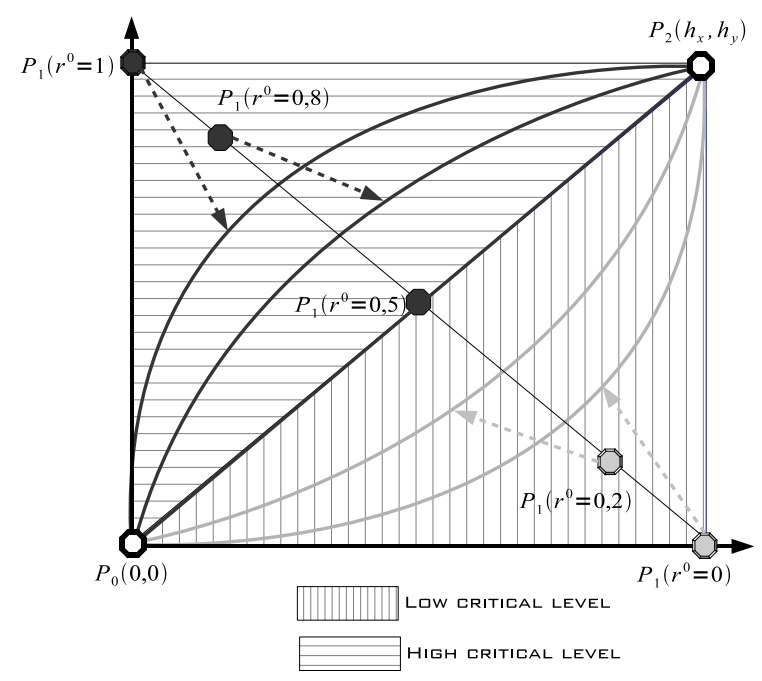

Fig. 3. The Behavior curve functions

3) The criticality level $r^{0}$ : As discussed above, the criticality level $r^{0}$ of an application is given into the interval $[0,1]$. According to this level, we define the criticality function called $\mathrm{Cr}$ which operates on the behavior point $P_{1}$ to control the $\mathrm{BV}$ function curvature.

According to the position of point $P_{1}$ the Bezier curve will morph between parabolic and hyperbolic form. As illustrated in figure 3 the first and the last points delimit the curve frame. This frame is a rectangle and is defined by the source point $P_{0}(0,0)$ and the threshold point $P_{2}\left(h_{x}, h_{y}\right)$. The middle point $P_{1}\left(b_{x}, b_{y}\right)$ controls the application criticality. We assume that this point can move through the second diagonal of the defined rectangle $b_{x}=\frac{-h_{y}}{h_{x}} * b_{y}+h_{y}$.

We define the $\mathrm{Cr}$ function as follows, such that varying $r^{0}$ between 0 and 1 gives updated positions for $P_{1}$ :

$$
\begin{gathered}
C r:[0,1] \quad \longrightarrow\left[0, h_{x}\right] *\left[0, h_{y}\right] \\
r^{0} \longrightarrow\left(b_{x}, b_{y}\right) \\
C r\left(r^{0}\right)=\left\{\begin{array}{l}
b_{x}=-h_{x} \times r^{0}+h_{x} \\
b_{y}=h_{y} \times r^{0}
\end{array}\right.
\end{gathered}
$$

Level $r^{0}$ is represented by the position of point $P_{1}$. If $r^{0}=0 P_{1}$ will have the coordinate $\left(h_{x}, 0\right)$. If $r^{0}=1 P_{1}$ will have the coordinate $\left(0, h_{y}\right)$.

\section{Resolving ambiguities}

Some applications such as surveillance and security applications, emergency detection in clinical environments and intrusion detection and tracking need more comprehensive interpretation of events or gestures. Access to multiple sources of visual data often allows for reducing ambiguities to allow for more accurate interpretation. Multiview has several advantages. First, the multi-view cameras can help circumvent occlusions. Second, even without occlusions, the information obtained from a single camera may be ambiguous for decision making, whereas a combination of information from multiple views may convey a higher confidence interpretation. Therefore, in our approach we allow collaboration among multiple cameras to reduce ambiguities, by adapting the activity nodes scheduling in a way to obtain more information about a target when it is necessary. In other words, to obtain multi-view of a target coming from more than one video node.

The problem studied here is known in the literature as the k-coverage problem [13]. Redundant sensing capabilities are usually required in sensor network applications for robustness, fault tolerance, or increased accuracy features. At the same time high sensor redundancy offers the possibility of increasing network lifetime by scheduling sleep intervals for some sensors and still providing continuous service with help of the remaining active sensors. In our approach, we use the k-coverage feature provided by the availability of multiple cover sets in order to reduce ambiguities by allowing video nodes to see the critical object from different perspectives.

When a node detects a critical event, it sends an urgent message to its neighbors to end the current round and begin a new one with a new scheduling scheme. The new scheduling must ensure that the target is covered by at least two or more video nodes. Therefore, from $C o(v)$, video node $v$ selects the cover that ensure the target's multi-coverage to be active. Then, it goes to sleep mode after sending its decision to its neighbors which in their turn schedule their activity, and a new round starts. This process is summarized in Algorithm 2.

\section{EXPERIMENTAL RESULTS}

To evaluate our approach we conducted a series of simulations based on the discrete event simulator OMNet++ [14]. Nodes have equal communication and sensing ranges of $30 \mathrm{~m}$ and $25 \mathrm{~m}$ respectively, an offset angle 


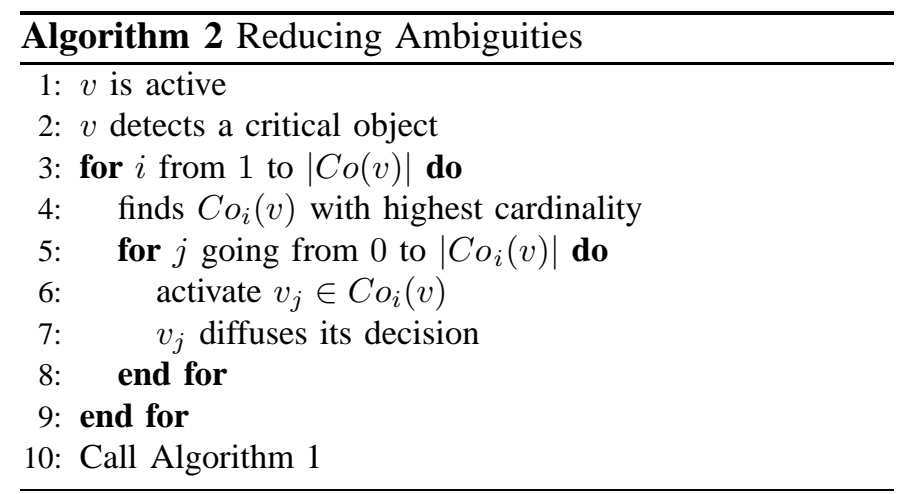

$\alpha$ of $\pi / 6$, a battery life of 100 units, random position $P$ and random direction $\vec{V}$. A simulation starts by a neighborhood discovery. Each node gathers positions and directions of its neighbors and finds the sets $A G, B G$ and $C G$. Then, round by round each node decides to be active or not. For these simulations, the round duration is set to 1s. Simulation ends as soon as the subset of nodes with power left is disconnected (when all active nodes have no neighbors anymore). We run each simulation 15 times to reduce the impact of randomness. Then, proportion of active nodes, area coverage and criticality impact were observed and analyzed.

\section{A. Coverage results}

In this section, we focus on the coverage results. We varied the deployed nodes density from 50 to 200 nodes in a $100 m * 100 m$ area. At the end of a round an active node decreases its battery life by one unit.

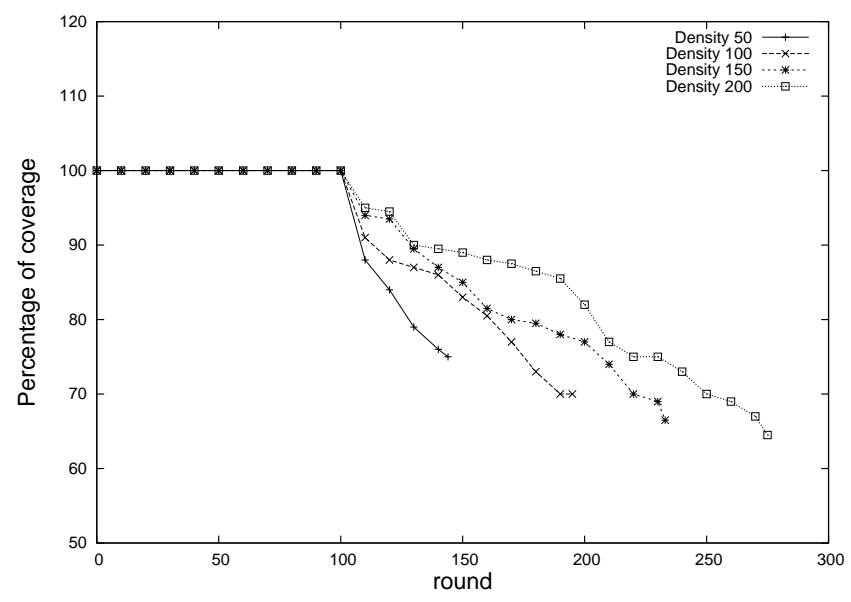

Fig. 4. Percentage of coverage.

The main objective of our approach is to maintain a full area coverage at each round. We define the full area coverage as the region covered initially by the whole network (i.e when all the deployed nodes are active). This area represents the union of all FoV areas of the deployed nodes. Figure 4 shows the percentage of area coverage round by round. This percentage is the ratio between the area covered by the set of active nodes over the initial covered area.

The initial sensing coverage is preserved for 100 rounds which is equal to a node's lifetime. At round 100, as expected, a set of nodes run out of energy. We can observe that in all the cases our algorithm guarantees a sensing coverage of at least $67 \%$ of the deployment area.

\section{B. Criticality impact}

For these set of experiments, we randomly deployed 150 sensor nodes in a $75 m * 75 m$ area. Each sensor node captures with a given number of frames per second (between 0fps and 6fps) in which case the battery capacity is decreased accordingly by 1 unit per captured frame (initial battery capacity is set to 100 units). The performance evaluation was realized with 2 fixed frame capture rate scenarios (3fps and 6fps) and 3 levels of application criticality that follows the dynamic rate model: $r^{0}=0$ (low criticality), $r^{0}=0.5$ and $r^{0}=1$ (high criticality) where a node's frame capture rate depends on the size of its cover set.

Figure 5 shows the percentage of coverage while varying the frame capture rate. Figure 6 shows the average frame capture rate of all active nodes per round which is representative of the surveillance application quality during the network lifetime.

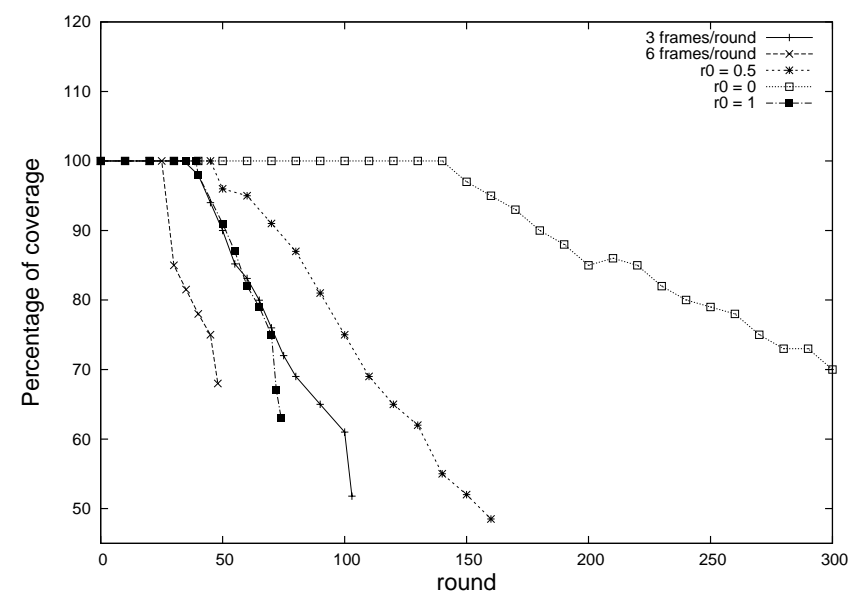

Fig. 5. Percentage of coverage while varying the frame capture rate.

As the application criticality $r^{0}$ is varied the frame capture rate of each sensor node that depends on its cover set size is modified according to the behavior function. Beyond the fact that our multiple levels activity model allows an application to specify a criticality level according to its objectives, the model also optimizes the area coverage as well as the network lifetime. For 


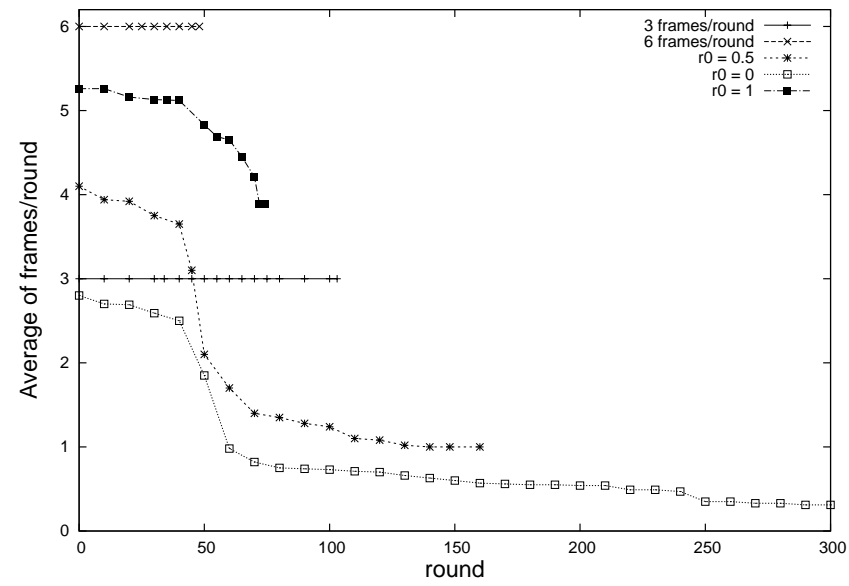

Fig. 6. Average frame capture rate per round.

instance, we can see in figure 6 that the high criticality scenario gives a mean frame capture rate of $4.63 \mathrm{fps}$ which should draw in figure 5 a curve that lies between the $6 \mathrm{fps}$ and the $3 \mathrm{fps}$ curves. However, figure 5 shows a high criticality curve very close to the 3 fps curve which indicates that our dynamic model can provide the same percentage of coverage but at a higher frame capture rate which should mean a better surveillance quality.

\section{Disambiguation feature}

To test how well our algorithm reduces the ambiguities, we consider a rectangular object which traverses the area of interest from left to right. The objective of this experiment is to determine the object trajectory and the identification time. In a surveillance application, faster the identification is, faster the interaction with the user is.

The rectangle as shown in Figure 7 is composed of 8 points, and is said to be fully identified when all 8 points are identified. In figure 7 , we observe that node $v_{1}$ can detect three points $\{a, d, h\}$, while node $v_{2}$ detects only $\{a\}$ (considering that point $\mathrm{a}$ hides $\mathrm{b}$ and $\mathrm{c}$ ) and $v_{3}$ sees $\{e, f\}$ ( $g$ is not well detected). In our simulation, $v_{2}$ and $v_{3}$ consider the object as critical, so the first node that detects the object enforces its neighbors to become active in order to have multiple views of the object.

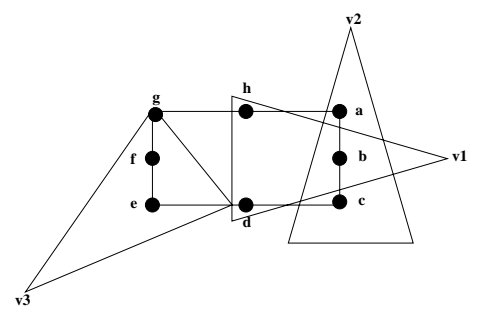

Fig. 7. The rectangular object in the region of deployment
The rectangular object $(4 m \times 2 m)$ traverses a $100 m *$ $100 \mathrm{~mm}$ area where we have randomly dispersed 150 video nodes. Then, we picked up the time taken by the network to identify the object, while varying its velocity. Figure 8 shows the variation of the identification time over the velocity of the object. We notice that this time is almost constant when using the ambiguity reduction scheduling. On the another hand, the identification takes a longer time in the case where just active nodes monitor the region.

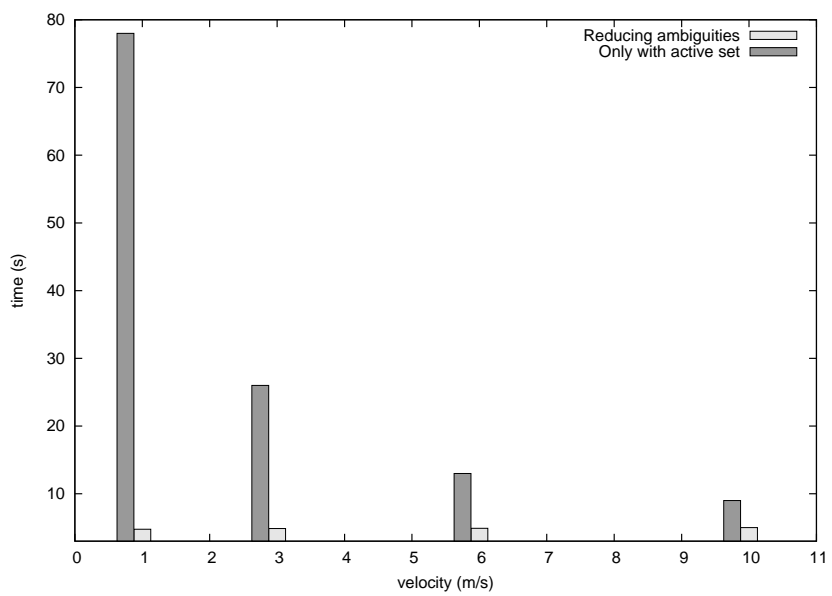

Fig. 8. The time taken by the network to identify the rectanglar object

\section{CONCLUSION}

Our contribution in this article is to provide the necessary algorithmic support for enabling critical video surveillance applications on WSN. Based on a simple coverage model that handle FoV redundancies by providing multiple covers per sensor node, we proposed a multiple levels activity model that uses behavior functions modeled by modified Bezier curves to define application classes and allow for adaptive scheduling. In addition, the availability of multiple cover sets then allows us to propose a simple but efficient disambigation algorithm. Besides providing a model for translating a subjective criticality level into a quantitative parameter of the surveillance system, our proposed approach for video sensor nodes can also optimize the resource usage by dynamically adjusting the provided service level. Future works will investigate how the risk factor in surveillance systems can be handled by our multiple levels activity model in order to automatically configure sensor nodes according to the environment stimulus.

\section{REFERENCES}

[1] C. Wang, M. T. Thai, Y. Li, F. Wang, and W. Wu, "Coverage based information retrieval for lifetime maximization in sensor networks," In IEEE GlobeCom, Nov. 2007. 
[2] J. Bahi, A. Makhoul, and A. Mostefaoui, "Hilbert mobile beacon for localization and coverage in sensor networks," International Journal of Systems Science, Taylor \& Francis, vol. 39, no. 11, pp. 1081-1094, Novembre 2008.

[3] A. Gallais, J. Carle, D. Simplot-Ryl, and I. Stojmenovic, "Localized sensor area coverage with low communication overhead," IEEE Transactions on Mobile Computing (TMC), vol. 7, no. 5, pp. 661-672, 2008.

[4] J. Bahi, A. Makhoul, and A. Mostefaoui, "Improving lifetime and coverage through a mobile beacon for high density sensor networks," The Second IEEE International Conference on Sensor Technologies and Applications, SENSORCOMM08, pp. 335-341, august 2008.

[5] Y. Cai, W. Lou, M. Li, and X.-Y. Li, "Target-oriented scheduling in directional sensor networks," 26th IEEE International Conference on Computer Communications, INFOCOM 2007, pp. 1550-1558, 2007.

[6] J. Ai and A. A. Abouzeid, "Coverage by directional sensors in randomly deployed wireless sensor networks," Journal of Combinatorial Optimization, vol. 11, no. 1, pp. 21-41, 2006.

[7] H. Liu, P. Wan, and X. Jia, "Maximal lifetime scheduling for sensor surveillance systems with k sensors to one target," IEEE Transactions on Parallel and Distributed Systems, vol. 17, no. 12, pp. 1526-1536, 2006.

[8] J. Wang, C. Niu, , and R. Shen, "Randomized approach for target coverage scheduling in directional sensor network," Lecture Notes in Computer Science - Springer, pp. 379-390, 2007.

[9] M. X. Cheng, L. Ruan, and W. Wu, "Achieving minimum coverage breach under bandwidth constraints in wireless sensor networks," in IEEE INFOCOM, 2005.

[10] H. Ma and Y. Liu, "Some problems of directional sensor networks," International Journal of Sensor Networks, vol. 2, no. 1-2, pp. 44-52, 2007.

[11] D. Tao, H. Ma, and L. Liu, "Coverage-enhancing algorithm for directional sensor networks," Lecture Notes in Computer Science - Springer, pp. 256-267, November 2006.

[12] R. Saadi, O. Hasan, J. Pierson, and L. Brunie, "Establishing trust beliefs based on a uniform disposition to trust," IEEE international Conference on Signal Image and Technologie Based Systems, 2007.

[13] W. Mo, D. Qiao, and Z. Wang, "Mostly-sleeping wireless sensor networks: Connectivity, k-coverage, and $\alpha$-lifetime," the Allerton Conference, UIUC, 2005.

[14] OMNeT++, "http://www.omnetpp.org/." 